\title{
CONSTRAINED POISSON ALGEBRAS AND STRONG HOMOTOPY REPRESENTATIONS
}

\author{
JIM STASHEFF
}

A Poisson algebra is a commutative associative algebra $A$ with an (anticommutative) bracket $\{$,$\} which is a derivation with respect to the commutative$ product: $\{f, g h\}=\{f, g\} h+f\{g, h\}$. Constraints constitute a distinguished set of elements $\phi_{\alpha}$ of $A$. They are said to be first class constraints if the ideal $I$ they generate (under the commutative product) is closed under Poisson bracket; $I$ need not be an ideal with respect to $\{$,$\} . This structure arises in$ physics with $A=C^{\infty}(W)$ for some symplectic manifold $W$. The constraints determine a subvariety $V \subset W$, the zero locus of $I$, and a foliation $\mathscr{F}$ of $V$, by the flows determined by the derivations $\{$,$\} . One wishes to compute the$ ad $I$-invariant functions on $V$, which would give $C^{\infty}(V / \mathscr{F})$ were the foliation to give a submersion $V \rightarrow V / \mathscr{F}$ onto a manifold.

In a remarkable series of papers, Fradkin, Batalin and Vilkovisky [0-3, 6] and then Henneaux [10] developed a method for calculating the ad $I$-invariant functions in $C^{\infty}(V)=A / I$ without passing through the quotient $A / I$. The method appeared to depend on solving certain specific, complicated equations and initially was applicable only locally and when $I$ was a regular ideal.

Using the techniques of 'homological perturbation theory' $[\mathbf{7}, \mathbf{8}, \mathbf{9}]$, I am able to justify their machinery in terms of the algebra alone, including, with Henneaux [11], the case of nonregular ideals [0]. The idea for this approach owes a great deal to the paper of Browning and McMullan [4], which revealed the structure of a multicomplex implicit in Fradkin et al and Henneaux.

The Lie algebra cohomology $H^{0}(I, A / I)$ computes the ad $I$-invariant functions on $V$, but physics requires a description in terms of $A$ and prefers to use $\Phi$, the linear span of the constraints $\phi_{\alpha}$, rather than the full ideal $I$. An obvious step algebraically is to replace $A / I$ by a free resolution over $A$. To combine this with the restriction to $\Phi \subset I$ is more subtle.

The Lie algebra cohomology of Cartan, Chevalley and Eilenberg [5] begins with the algebra $\operatorname{Alt}(I, A / I)$ of alternating multilinear functions on $I$ with values in $A / I$ and a differential Alt $\rightarrow$ Alt (which increases the number of variables by one) given in terms of the bracket on $I$ and the adjoint representation of $I$ on $A / I$ : For example, for $h: I \rightarrow A$, we have

$$
(\delta h)(f, g)=h(\{f, g\})-\{f, h(g)\}+\{g, h(f)\} \text {. }
$$

The subalgebra $\operatorname{Alt}_{A}(I, A / I)$ of $A$-multilinear functions is in fact a subcomplex with the same $H^{0}$. (This is isomorphic to the complex which defines

Received by the editors July 1, 1987 and, in revised form, November 23, 1987.

1980 Mathematics Subject Classification (1985 Revision). Primary 18G10, 17B55, 81E13; Secondary 58H10, 70H99, $81 \mathrm{C} 99$.

Research supported by the NSF and the Institute for Advanced Study. 
the Rinehart cohomology of the $(A / I, R)$-Lie algebra $I / I^{2}$ with coefficients in $A / I[12]$.$) The inclusion \Phi \subset I$ induces $\operatorname{Alt}_{A}(I, A / I) \rightarrow \operatorname{Alt}(\Phi, A / I)$ and a differential also denoted $\delta$. (This map is an isomorphism if $I$ is regular.)

Now introduce a multiplicative resolution $\pi: K_{I} \rightarrow A / I$, that is, $K_{I}$ is a graded commutative differential algebra (with differential $d$ ) and $\pi$ induces an isomorphism $\pi^{*}: H_{0}\left(K_{I}\right) \rightarrow A / I$ with $H_{i}\left(K_{I}\right)=0$ otherwise. For example, if $I$ is a regular ideal, take $K_{I}$ to be the Koszul complex; more generally, the Tate resolution will do [14]. If we replace $A / I$ by $K_{I}$ and consider $\operatorname{Alt}\left(\Phi, K_{I}\right)$, the problem is to extend $d$ to a differential $D$ so as to realize the same homology as that of $\operatorname{Alt}(\Phi, A / I)$ with respect to $\delta$. The major source of difficulty is that the adjoint representation of $I$ on $A / I$ does not lift to $K_{I}$; in spite of this, we have:

THEOREM 1. There are differentials $\delta_{i}$ on $\operatorname{Alt}\left(\Phi, K_{I}\right)$ which increase the form degree by $i$ and the resolution degree by $i-1$ such that $\delta_{0}=d$ and $D=\sum \delta_{i}$ has $D^{2}=0$ with $\pi: K_{I} \rightarrow A / I$ inducing

$$
H^{0}\left(\operatorname{Alt}\left(\Phi, K_{I}\right), D\right) \approx H^{0}(\operatorname{Alt}(I, A / I), \delta) .
$$

Our proof of the theorem uses the methods of homological perturbation theory $[\mathbf{7}, \mathbf{8}, 9]$. Let $\operatorname{Der}^{q} K$ denote the derivations of $K_{I}$ which increase resolution degree by $q$. The collection Der $K_{I}=\left\{\operatorname{Der}^{q} K_{I}\right\}$ is made into a differential graded Lie algebra by using the graded commutator of derivations and the induced differential: $d \theta=[d, \theta]$. We cannot, in general, find a representation of $I$ in Der $K_{I}$, but we can find a "strong homotopy representation", meaning a family $\Theta_{i} \in \operatorname{Alt}^{i}\left(I, \operatorname{Der} K_{I}\right)$ for $i \geq 1$ satisfying the following relations: For $i=1, \Theta^{1}(f)=\{f$,$\} . For i>1$, and $\bar{f}_{i}=\left(f_{0}, \ldots, f_{i}\right)$,

(*) $\left[d, \Theta^{i}\right] \bar{f}_{i}=\sum\left[\Theta^{j}, \Theta^{k}\right]\left(\bar{f}_{i}\right)+\sum(-1)^{j+k} \Theta^{i-1}\left(\left\{f_{j}, f_{k}\right\}, \ldots, \hat{j}, \ldots, \hat{k}, \ldots\right)$.

For $i=1$, this is to be interpreted as $\left[d, \theta^{1}\right]=0$. Here $[$,$] is the usual$ induced bracket on $\operatorname{Alt}(V, L)$ for a vector space $V$ and Lie algebra $L$. The maps $\Theta^{i}$ are constructed inductively, using a contracting homotopy $s$ for $K_{I}$, that is: $s d+d s=1-\bar{\pi}$ where $\bar{\pi}: K_{I} \rightarrow A / I \rightarrow A \hookrightarrow K_{I}$ and the map $1-\bar{\pi}$ is the identity on $I$. We begin by defining $\Theta^{1}: I \rightarrow \operatorname{Der}^{0} K_{I}$ as an extension of the adjoint action of $I$ on $A$ as follows: By induction on the resolution degree of a generator $x$ of $K_{I}$ over $A$, define $\Theta^{1}(f)(x)=s \Theta^{1}(f)(d x)$. Verify directly that $(*)$ is valid in the form $\left[d, \Theta^{1}\right]=0$. Now assume we have constructed $\Theta^{i}$ for $i<n$ to satisfy (*). Let RHS denote the right-hand side of the equation $(*)$ for $i=n$. Verify that $[d, \mathrm{RHS}]=0$ using $(*)$ and the Jacobi identity. Now define the derivation $\Theta^{n}\left(\bar{f}_{n}\right)$ as $s$ RHS. We verify that

$$
\begin{aligned}
{[d, s \mathrm{RHS}] } & =d s \mathrm{RHS}+s \mathrm{RHS} d=(d s+s d) \mathrm{RHS} \text { by induction } \\
& =(1-\bar{\pi}) \mathrm{RHS}=\mathrm{RHS},
\end{aligned}
$$

since RHS raises resolution degree by at least $j-1+k-1$, which is into the kernel of $\pi$ unless $j=k=1$. For $n=2$, we also use the fact that $\Theta^{1}$ is an extension of the adjoint action of $I$ on $A$ in terms of the original Poisson bracket. 
Since $\Phi \subset I$ need not be closed under the bracket, we cannot just restrict $D$ to $\operatorname{Alt}\left(\Phi, K_{I}\right)$. Instead, the FBV construction in the regular case makes further use of the Poisson algebra. Notice that the Koszul resolution can be written as $A \otimes \bigwedge s \Phi$ where $s \Phi$ is isomorphic to $\Phi$ as a vector space, while $\operatorname{Alt}\left(\Phi, K_{I}\right)$ contains the vector space dual $\Phi^{*}=\operatorname{Hom}(\Phi, R)$. Extend the Poisson bracket of $A$ to all of $\operatorname{Alt}\left(\Phi, K_{I}\right)$ by first defining $\left\{\Phi^{*}, s \Phi\right\}$ to be isomorphic to the usual dual pairing and then extending to a graded Poisson bracket by using the derivation property: $\{\omega, \eta \wedge \varsigma\}=\{\omega, \eta\} \wedge \varsigma+(-1)^{|\omega||\eta|} \eta \wedge$ $\{\omega, \varsigma\}$.

THEOREM 2. There is an element $Q \in \prod \operatorname{Alt}^{p}\left(\Phi, K_{I}\right)$ such that $D$ in Theorem 1 is given by $D=\{Q$,$\} .$

We write $Q=\sum Q_{p}$ where $Q_{p} \in \operatorname{Alt}^{p+1}\left(\Phi, K_{I}\right)$ takes values in $A \otimes \bigwedge^{p} s \Phi$. Although $D=\{Q$,$\} , we do not have \delta_{i}=\left\{Q_{i},\right\}$ but rather $\delta_{i}$ is of bidegree $(i, i-1)$, while $\{Q$,$\} has components of bidegree (i, i-1)$ and $(i+1, i)$. To start, let $Q_{0}$ be the inclusion $\iota: \Phi \hookrightarrow A \hookrightarrow K_{I}$ so that $\left\{Q_{0},\right\} \mid K_{I}$ is the Koszul differential. (This is easier to see in terms of a basis $\left\{\phi_{\alpha}\right\}$ for $\Phi$, dual basis $\left\{\eta^{\alpha}\right\}$ for $\Phi^{*}$, and basis $\left\{\mathscr{P}_{\alpha}\right\}$ for $s \Phi$ so that $Q_{0}=\phi_{\alpha} \eta^{\alpha}$.) Filter $\operatorname{Alt}\left(\Phi, K_{I}\right)$ by $F^{p}=\sum_{i \leq p} \mathrm{Alt}^{i}$, and for any element $R$ of the complex, let $R^{2}$ denote $\frac{1}{2}\{R, R\}$. Now construct $Q_{i}$ by induction so that the partial sums $R_{i}=\sum Q_{j}$ have the following properties:

$$
R_{p}^{2} \in F^{p+2} \quad \text { and } \quad d R_{p}^{2} \in F^{p+3} .
$$

Define $Q_{n+1}=-s R_{n}^{2}$. A slightly complicated computation then shows that $R_{n+1}$ satisfies the inductive hypothesis.

We have left to show that $D$ gives the desired homology. The resolution $\pi: K_{I} \rightarrow A / I$ induces a map of complexes. If we filter $\operatorname{Alt}\left(\Phi, K_{I}\right)$ as above, the associated graded has differential just $d$ with homology $\operatorname{Alt}(\Phi, A / I)$. A standard spectral sequence argument then gives the desired result.

Because of the motivating physics, Fradkin et al consider also the situation in which $A$ is a super-Poisson algebra, i.e. $\mathbf{Z} / 2$-graded with appropriate signs throughout. Now we need to use a super-resolution, for example, Jozefiak's [13]. The formalism we have used need only be made super (i.e. attend carefully to signs) with some extra care interpreting formal power series.

As a guide to the physics literature, in the regular case, $Q_{i}$ corresponds to an expression $U_{\underline{\beta}}^{\bar{\alpha}} \eta \underline{\beta}_{\bar{\alpha}}$ where $\bar{\alpha}=\alpha_{1} \cdots \alpha_{i-1}, \underline{\beta}=\beta_{1} \cdots \beta_{i+1}$ and $\eta \underline{\underline{\beta}}=$ $\eta^{\beta_{1}} \wedge \cdots \wedge \eta^{\beta_{i+1}}$, etc. Finally, the $\eta^{\beta}$ are called ghosts, the $\mathscr{P}_{\alpha}$ anti-ghosts and, in the nonregular case, syzygies are called extraghosts or ghosts-of-ghosts-of-

\section{REFERENCES}

0. I. A. Batalin and E. S. Fradkin, A generalized canonical formalism and quantization of reducible gauge theories, Phys. Lett. 122B (1983), 157-164.

1. I. A. Batalin and G. S. Vilkovisky, Existence theorem for gauge algebra, J. Math. Phys. 26 (1985), 172-184.

2. , Quantization of gauge theories with linearly dependent generators, Phys. Rev. D 28 (1983), 2567-2582. 
3. _ Relativistic S-matrix of dynamical systems with boson and fermion constraints, Phys. Lett. 69B (1977), 309-312.

4. A. D. Browning and D. McMullan, The Batalin, Fradkin, Vilkovisky formalism for higher order theories, J. Math. Phys. 28 (1987), 438-444.

5. C. Chevalley and S. Eilenberg, Cohomology theory of Lie groups and Lie algebras, Trans. Amer. Math. Soc. 63 (1948), 85-124.

6. E. S. Fradkin and G. S. Vilkovisky, Quantization of relativistic systems with constraints, Phys. Lett. 55B (1975), 224-226.

7. V. K. A. M. Gugenheim, On a perturbation theory for the homology of a loop space, J. Pure Appl. Algebra 25 (1982), 197-205.

8. V. K. A. M. Gugenheim and J. P. May, On the theory and application of torsion products, Mem. Amer. Math. Soc. No. 142, Amer. Math. Soc., Providence, R. I., 1974.

9. V. K. A. M. Gugenheim and J. D. Stasheff, On perturbations and $A_{\infty}$-structures, Bull. Soc. Math. Belg. 38 (1986), 237-245.

10. M. Henneaux, Hamiltonian form of the path integral for theories with a gauge freedom, Phys. Rep. 126 (1985), 1-66.

11. M. Henneaux and J. Stasheff, BRST formalism for reducible theories (preprint).

12. G. Rinehart, Differential forms for general commutative algebras, Trans. Amer. Math. Soc. 108 (1963), 195-222.

13. T. Jozefiak, Tate resolutions for commutative graded algebras over a local ring, Fund. Math. 74 (1972), 204-231.

14. J. Tate, Homology of Noetherian rings and local rings, Illinois J. Math. 1 (1957), 14-27.

Department of MAThematics, University of NoRTh Carolina, Chapel HILL, NORTH CAROLINA 27514 